\title{
Point mutations in $w c h A$ are responsible for the non-typability of two invasive Streptococcus pneumoniae isolates
}

\author{
Sara Melchiorre, ${ }^{1} \dagger$ Romina Camilli, ${ }^{2} \dagger$ Agostina Pietrantoni, ${ }^{3}$ \\ Monica Moschioni, ${ }^{1}$ Francesco Berti, ${ }^{1}$ Maria Del Grosso, ${ }^{2}$ \\ Fabiana Superti, ${ }^{3}$ Michèle Anne Barocchi ${ }^{1}$ and Annalisa Pantosti ${ }^{2}$ \\ ${ }^{1}$ Novartis Vaccines \& Diagnostics, Siena, Italy \\ ${ }^{2}$ Department of Infectious, Parasitic and Immune-Mediated Diseases, Istituto Superiore di Sanità, \\ Rome, Italy \\ ${ }^{3}$ Department of Technology and Health, Istituto Superiore di Sanità, Rome, Italy
}

Correspondence

Annalisa Pantosti

annalisa.pantosti@iss.it

Received 25 August 2011

Revised 14 October 2011

Accepted 24 October 2011

\begin{abstract}
Non-typable Streptococcus pneumoniae (NTPn) strains are typically isolated from nasopharyngeal carriage or from conjunctivitis. Since the isolation of NTPn from invasive disease is rare, we characterized the genetic basis of the non-typability of two isolates obtained in Italy from two cases of bacteraemic pneumonia. MLST revealed that both NTPn belonged to ST191, which, according to the MLST database, is associated with serotype 7F. Sequencing of the capsular locus (cps) confirmed the presence of a 7F cps in both strains and revealed the existence of distinct single point mutations in the $w c h A$ gene (a glycosyltransferase), both leading to the translation of proteins truncated at the $C$ terminus. To verify that these mutations were responsible for the non-typability of the isolates, a functional 7F WchA was overexpressed in both NTPn. The two NTPn along with their WchA-overexpressing derivatives were analysed by transmission electron microscopy and by high-resolution magic angle spinning NMR spectroscopy. Both NTPn were devoid of a polysaccharide capsule, and WchA overexpression was sufficient to restore the assembly of a serotype 7F capsule on the surface of the two NTPn. In conclusion, we identified two new naturally occurring point mutations that lead to non-typability in the pneumococcus, and demonstrated that WchA is essential for the biosynthesis of the serotype 7F capsule.
\end{abstract}

\section{INTRODUCTION}

The Gram-positive pathogen Streptococcus pneumoniae is a major cause of community-acquired pneumonia, as well as upper respiratory tract infections such as acute otitis media and sinusitis, and invasive diseases such as meningitis, bacteraemia and endocarditis. However, S. pneumoniae is also a commensal of the upper respiratory tract, especially of young children, who represent the reservoir for pneumococcal transmission within the community.

The ability of the pneumococcus to invade sterile sites is related to the expression of a polysaccharide capsule, which

†These authors contributed equally to this work/paper.

Abbreviations: CPS, capsular polysaccharide; HR-MAS NMR, highresolution magic angle spinning NMR; NTPn, non-typable Streptococcus pneumoniae; TEM, transmission electron microscopy.

A supplementary table, showing oligonucleotides used to amplify and sequence the 7F capsular locus, is available with the online version of this paper. provides a barrier against host cell-mediated phagocytosis and allows bacterial persistence in the blood (Brown et al., 1983). In addition, capsular polysaccharides (CPSs) are immunogenic and antibodies against CPSs provide protection against pneumococcal disease; therefore, all of the current pneumococcal vaccines are based on a combination of different CPSs, either unconjugated or conjugated to a carrier protein (Gladstone et al., 2011).

To date, 93 capsular types (serotypes) have been described on the basis of their different genetic, biochemical and antigenic properties (Bentley et al., 2006; Calix \& Nahm, 2010; Park et al., 2007). Each pneumococcal serotype corresponds to a distinct CPS, synthesized on the bacterial surface by enzymes which are encoded within a single capsule locus ( $c p s)$, with a few notable exceptions (Llull et al., 1999). The sequencing of different $c p s$ loci has revealed the presence of a core set of genes common to all capsular types, namely $w z g, w z h, w z d$ and wze (also known as $c p s A, B, C$ and $D$ ), and of additional genes that are CPS-specific (Bentley et al., 2006). 
The different capsular types can be distinguished in the laboratory by reaction with type-specific antisera, evidenced by the capsular swelling or Quellung reaction (Austrian, 1976). Only a minority of S. pneumoniae strains do not react with the anticapsular antisera; these strains, defined as non-typable Streptococcus pneumoniae (NTPn), are thought to be non-encapsulated. NTPn are typically isolated from carriage (Andrade et al., 2010; Sá-Leão et al., 2006), but are also found in conjunctivitis (Carvalho et al., 2003), acute otitis media (Xu et al., 2011) and, rarely, in invasive disease (Beall et al., 2006). Non-typability can be due to different genetic modifications, such as partial or complete loss of the cps gene cluster (Hathaway et al., 2004), and the presence of a novel gene (Baldry et al., 2009), single point mutations (Arrecubieta et al., 1994) or sequence duplications (Waite et al., 2001).

The characterization of NTPn by MLST has revealed the presence of specific non-typable pneumococcal lineages which have lost the cps gene cluster (Hanage et al., 2006; Hathaway et al., 2004) as well as of NTPn that are genetically related to encapsulated strains (Andrade et al., 2010; Hathaway et al., 2004).

In this report, we investigated the genetic basis for nontypability of two NTPn isolates responsible for invasive disease. The strains were isolated in the same Italian hospital during 2006 through the surveillance network of invasive pneumococcal disease (Gherardi et al., 2009) from two cases of bacteraemic pneumonia.

\section{METHODS}

Bacterial strains and growth conditions. S. pneumoniae AP422 and AP426 were isolated from the blood of two adult patients with pneumonia admitted to the same Italian hospital in 2006. S. pneumoniae AP425 (clinical isolate, serotype 7F) was used as a control. Strains were grown at $37{ }^{\circ} \mathrm{C}$ in a $5 \% \mathrm{CO}_{2}$-enriched atmosphere on Columbia agar plates (Oxoid) with $5 \%$ sheep blood, or on Tryptic Soy Agar plates (Becton Dickinson) supplemented with $10 \mathrm{mg}$ colistine $\mathrm{l}^{-1}, 5 \mathrm{mg}$ oxolinic acid $\mathrm{l}^{-1}$ and $5 \%$ sheep blood. For genomic DNA extraction and NMR spectroscopy, liquid cultures were grown statically at $37{ }^{\circ} \mathrm{C}$ in $5 \% \mathrm{CO}_{2}$ in Todd-Hewitt broth (Becton Dickinson) supplemented with $0.5 \%$ (w/w) yeast extract (THYE) to $\mathrm{OD}_{600} 0.25$.

Serotyping. Serotyping was performed by the latex agglutination test and the Quellung reaction, using antisera produced by the Statens Serum Institute (Copenhagen, Denmark).

MLST. Genomic DNA from pneumococcal strains was prepared with the Wizard Genomic DNA Purification kit (Promega) following the manufacturer's instructions. MLST was performed as previously described (Bagnoli et al., 2008).

7F cps detection and sequencing. The amplification and sequencing of 7F cps was performed on the genomic DNA of strains AP422, AP426 and AP425 using the primers listed in Supplementary Table S1, generating short overlapping DNA fragments. Amplicons were purified with magnetic carboxylated beads (Agencourt Bioscience); sequencing was carried out with an ABI 3730xl DNA analyser (Applied Biosystems). Traces were visually inspected, edited, assembled and analysed with Vector NTI Advance 10.3.1 (Life Technologies).

Cloning and S. pneumoniae transformation. The shuttle plasmid pMU1328_Pc_wchA was obtained as described below. Briefly, the wchA gene of the AP425 strain and the constitutive promoter of erythromycin $(P c)$ (Gentile et al., 2011) were amplified with the primers wchA_for (5'-GTGCGTGGATCCATGGATGAAAAAGGATTGAAAATT- $\left.3^{\prime}\right)$ and $w c h A \_r e v\left(5^{\prime}\right.$-CAGCGTGGATCCTCACTTCGCCCCTTCTCTCATAAA- $\left.3^{\prime}\right)$, and $P c_{-}$for $\left(5^{\prime}\right.$-GTGCGTGAATTCGAAACAGCAAAGAATGGCGGAAAC-3') and $P c_{-}$rev $\left(5^{\prime}\right.$-CAGCGTGGATCCGTAATCACTCCTTCTTAATTACAA-3'), respectively. The two PCR products, digested with BamHI and EcoRI/BamHI restriction enzymes, respectively, were cloned into pMU1328, containing an erythromycin-resistance marker (Achen et al., 1986). The ligation mixtures were then transformed into competent Escherichia coli DH10B, and transformants were selected on plates supplemented with erythromycin $\left(100 \mu \mathrm{g} \mathrm{ml}^{-1}\right) . P c_{-} w c h A$ insertion was confirmed by sequencing. pMU1328_Pc_wchA was then transformed into AP422 and AP426 by conventional methods (Alloing et al., 1998), and transformants were selected using erythromycin $\left(1 \mu \mathrm{g} \mathrm{ml}^{-1}\right)$, analysed by PCR to confirm the presence of the plasmid, and further investigated for the presence of the capsule.

Transmission electron microscopy (TEM). For ultrastructural analysis of the polysaccharide capsule, bacterial strains were grown on Columbia agar plates and prepared following the immune-stabilization method (Jacques \& Gottschalk, 1997). Briefly, bacteria were resuspended in $0.5 \mathrm{ml} \mathrm{PBS}$, pH 7.4, and incubated with $0.1 \mathrm{ml}$ type 7 antiserum (Statens Serum Institute) for $1 \mathrm{~h}$ at $4{ }^{\circ} \mathrm{C}$. After immunestabilization, bacterial cells were centrifuged at $2500 \mathrm{~g}$ for $10 \mathrm{~min}$ at $4{ }^{\circ} \mathrm{C}$, and fixed in fixation solution containing $2.5 \%(\mathrm{v} / \mathrm{v})$ glutaraldehyde, $0.075 \mathrm{M}$ lysine acetate in cacodylate buffer $(0.1 \mathrm{M}$, $\mathrm{pH} 7.0)$ with $0.075 \%(\mathrm{w} / \mathrm{v})$ ruthenium red (CR buffer) for $2 \mathrm{~h}$ at $4{ }^{\circ} \mathrm{C}$. Controls processed using an unrelated antiserum (type 21), or without antiserum stabilization, were performed simultaneously. All bacterial samples were then washed three times with CR buffer and post-fixed with $1 \%(\mathrm{w} / \mathrm{v})$ osmium tetroxide in CR buffer for $1 \mathrm{~h}$ at room temperature. Subsequently, samples were washed three times with cacodylate buffer, dehydrated through a graded series of ethanol, and embedded in Agar 100 epoxy resin (Agar Scientific). Ultrathin sections of bacterial samples were placed on Formvar-coated copper grids, post-stained with uranyl acetate and lead citrate, and examined with a Philips 208s electron microscope at $80 \mathrm{kV}$.

Type 7F CPS detection by high-resolution magic angle spinning NMR spectroscopy (HR-MAS NMR). Bacteria recovered from THYE were inactivated by $1 \%(\mathrm{v} / \mathrm{v})$ formaldehyde treatment and then washed three times with PBS in deuterium oxide $\left(\mathrm{D}_{2} \mathrm{O}\right.$; Sigma-Aldrich). Approximately $50 \mu \mathrm{l}$ of compact pellet was inserted into a Kel-F disposable insert for a $50 \mu \mathrm{l}$ volume and then in a $4 \mathrm{~mm}$ MAS $\mathrm{ZrO}_{2}$ rotor (Bruker). Proton HR-MAS NMR experiments were recorded by a Bruker Avance III $400 \mathrm{MHz}$ spectrometer using a Bruker $4 \mathrm{~mm}$ HR-MAS probe. The spectra were recorded at a $4500 \mathrm{~Hz}$ spin rate and $25{ }^{\circ} \mathrm{C}$. The ${ }^{1} \mathrm{H}$ spectra were acquired with the combination of a diffusion filter pulse sequence with gradient pulses (diffusion filter $85 \%$ ), to remove the low-molecular-mass species free in solution, and a Carr-Purcell-Meiboom-Gill (CPMG) pulse sequence $\left[90-(\tau-180-\tau)_{n}\right.$-acquisition] as T2 filter $(76.8 \mathrm{~ms})$, to remove the broad signals of larger molecular species. Five milligrams of purified 7F polysaccharide (Merck) were solubilized in $0.75 \mathrm{ml} \mathrm{D} \mathrm{D}_{2} \mathrm{O}$ (Sigma-Aldrich), the solution was inserted into a $5 \mathrm{~mm}$ NMR tube (Wilmad), and the proton NMR experiment was recorded at $25^{\circ} \mathrm{C}$ by the same spectrometer, using a $5 \mathrm{~mm}$ broadband probe (Bruker). The ${ }^{1} \mathrm{H}$ NMR spectrum was recorded using a standard one-pulse experiment. Both the solid- and liquid-state NMR spectra were collected with $32 \mathrm{k}$ data points over a 10 p.p.m. spectral width. The 
transmitter was set at the water resonance frequency, which was also used as the reference signal (4.79 p.p.m.). The TopSpin 2.1 software package (Bruker) was used for data acquisition and processing of all spectra.

\section{RESULTS}

\section{NTPn strains AP422 and AP426 contain a 7F capsular locus with point mutations in wchA}

The two invasive S. pneumoniae strains, AP422 and AP426, were defined as non-typable, since serotype testing (confirmed by the Statens Serum Institute) did not reveal positivity for any capsular type. In order to verify that these strains belonged to a clonal lineage specifically referable to non-typable pneumococci, they were subjected to MLST analysis. Both AP422 and AP426 were ST191, which, according to a search performed on the $S$. pneumoniae MLST database (http://www.mlst.net/, accessed on 18 August 2011), is mainly (96\%) associated with serotype 7F. Using PCR amplification, 7F cps was detected in both AP422 and AP426 and then sequenced. Comparison with two reference 7F cps sequences (GenBank accession nos CR931643 and ABFT01000009) revealed the presence of the entire 7F locus, with an overall nucleotide identity of $99.8 \%$ in the two strains. Sequence analysis highlighted the existence of a distinct single point mutation in the wchA gene in both strains. With respect to the reference wch $A$ nucleotide sequence, AP422 wchA showed a frameshift mutation due to the deletion of an adenine in position 910, while AP426 wchA showed a G1081T transversion, which creates a new stop codon. Both mutations lead to the formation of a shorter wchA gene associated with the translation of a protein truncated at the $\mathrm{C}$ terminus (Fig. 1). The NCBI Conserved Domain algorithm (http://www. ncbi.nlm.nih.gov/cdd) predicts the WchA catalytic domain to be at the $\mathrm{C}$ terminus of the protein (Fig. 1), so that the truncated WchA proteins encoded by AP422 and AP426 are predicted to be enzymically inactive. In the polysaccharide biosynthesis pathway, WchA (also known as CpsE) is the initial glucose phosphate-transferase, responsible for the linkage of an activated glucose phosphate to the lipid carrier (Pelosi et al., 2005). Since WchA has been demonstrated to be required for the biosynthesis of other capsular types (Pelosi et al., 2005), the mutations identified in wchA in both AP422 and AP426 are likely to be responsible for their non-typability.

\section{Overexpression of a functional WchA restores the ability of $S$. pneumoniae to synthesize the $7 F$ capsule}

With the aim of demonstrating that the mutations present in wchA were sufficient to account for the lack of capsule in AP422 and AP426, a functional WchA was overexpressed in both NTPn strains. AP422 and AP426 were transformed with the recombinant plasmid pMU1328_Pc_wchA to generate the respective recombinant strains $A P 422 \nabla \mathrm{p}$ MU1328_Pc_wchA and AP426DpMU1328_Pc_wchA. The two transformed NTPn yielded a positive reaction with the 7F antiserum in the Quellung reaction. To confirm that WchA overexpression had restored the ability to synthesize a capsule at levels comparable with those of a wild-type capsulated strain, the NTPn isolates along with their WchAoverexpressing derivatives were analysed by TEM and by HR-MAS NMR, using the serotype 7F strain AP425 as a positive control. For TEM analysis, the two NTPn strains along with AP425 and the WchA-overexpressing derivatives were stabilized with type 7 antiserum before processing for TEM observation. This method was chosen as an alternative

\footnotetext{
$554 / 621$ MDEKGLKIFLAVLQSIIVILLVYFLSFVREAELERSSMVILYLLHFFVFYFSSYGNNFFKRGYLVEFNSTIRYIFFFAIAISVLNFFIAERFSISRRGMV AP 422 1 MDEKGLKIFLAVLQSIIVILLVYFLSFVREAELERSSMVILYLLHFFVFYFSSYGNNFFKRGYLVEFNSTIRYIFFFAIAISVLNFFIAERFSISRRGMV AP426 1 MDEKGLKIFLAVLQSIIVILLVYFLSFVREAELERSSMVILYLLHFFVFYFSSYGNNFF KRGYLVEFNSTIRY IFFFAIAISVLNFFIAERFSISRRGMV

554/62 101 YFLTLEGISLYLLNFLVKKYWKHVFFNLKNSKKILLLTVTKNMEKVLDKLLESDELSWKLVAVSVLDKSDFQHDKIPVIEKEKIIEFATHEVVDEVFVNL AP422 101 YFLTLEGISLYLLNFLVKKYWKHVFFNLKNSKKILLLTVTKNMEKVLDKLLESDELSWKLVAVSVLDKSDFQHDKIPVIEKEKIIEFATHEVVDEVFVNL AP426 101 YFLTLEGISLYLLNFLVKKYWKHVFFNLKNSKKILLLTVTKNMEKVLDKLLESDELSWKLVAVSVLDKSDFQHDKIPVIEKEKIIEFATHEVVDEVFVNL

554/62 201 PGESYDIGEIISRFETMGIDVTVNLKAFDKNLGRNKQIYEMVGLNVVTFSTNFYKTSHVISKRILDICGATIGLILFAIASLVLVPLIRKDGGPAIFAQT AP422 201 PGESYDIGEIISRFETMGIDVTVNLKAFDKNLGRNKQIYEMVGLNVVTFSTNFYKTSHVISKR ILDICGATIGLILFAIASLVLVPLIRKDGGPAIFAQT AP426 201 PGESYDIGEIISRFETMGIDVTVNLKAFDKNLGRNKQIYEMVGLNVVTFSTNFYKTSHVISKRILDICGATIGLILFAIASLVLVPLIRKDGGPAIF AQT

554/62 301 RIGKNGRHF TFYKFRSMRSDAEAIKEQLMDQNTMQGGMFKIDNDPRVTK IGRF IRKTSLDELPQFWNVF IGDMSLVGTRPPTVDEYVQYTPEQKRRLSFK AP422 301 RIGKMVDILPFINSVRCGAMLKLSKNS

AP426 301 RIGKNGRHFTFYKFRSMRSDAEAIKEQLMDQNTMQGGMFKIDNDPRVTKIGRF IRKTSLD
}

554/62 401 PGITGLWQVSGRSKITDFDDVVKLDVAYIDNWTIWKDIE ILLKTVKVVFMREGAK

Fig. 1. Alignment of the WchA amino acid sequences of the reference strain 554/62 (GenBank accession no. CR931643) and the two NTPn, AP422 and AP426. In both AP422 and AP426, wchA is translated as a C-terminal-truncated protein. Bold type indicates the catalytic domain as predicted by the NCBI Conserved Domain algorithm; italic type indicates an alternative C terminus due to a frameshift in the nucleotide sequence of AP422. 
to the standard fixation protocol with lysine and ruthenium red (Kreikemeyer et al., 2011); indeed, the latter appeared to be inadequate to visualize the $7 \mathrm{~F}$ capsule polysaccharide (data not shown), since it does not contain anionic charged moieties (Moreau et al., 1988). After immune-stabilization, a well-preserved dense and thick capsular material was observed surrounding the AP425 bacterial cells (Fig. 2a), while non-typable AP422 (Fig. 2b) and AP426 (Fig. 2c) did not exhibit the characteristic polysaccharide layer on the surface. In WchA-overexpressing AP422 and AP426 (Fig. 2d, e), TEM examination revealed cells surrounded by a CPS structure comparable with that of AP425, although the capsule of WchA-overexpressing AP422 appeared heterogeneous and in some bacterial cells slightly irregular and thinner. In order to confirm the data obtained by TEM, proton HR-MAS NMR spectra directly recorded on inactivated cells in the heterogeneous phase were obtained. ${ }^{1} \mathrm{H}$ NMR spectra collected on purified polysaccharide in the liquid state clearly revealed specific peaks corresponding to the 7F CPS, such as the anomeric protons at 5.0-5.6 p.p.m. and the C6 methyl group of rhamnose at 1.2-1.4 p.p.m. (Fig. 3a, b). Spectral analysis showed the absence of the 7Fspecific peaks in the two NTPn and in AP422 and AP426 transformed with an empty pMU1328 plasmid (Fig. 3b), while the above-mentioned 7F-specific peaks were present both in the capsulated AP425 and in the WchA-overexpressing AP422 and AP426 (Fig. 3b). Although signals of other ${ }^{1} \mathrm{H}$-NMR-sensitive molecules expressed on the bacterial surface partially overlapped with the CPS peaks, the anomeric proton at 5.6 p.p.m. and the C6 methyl group of rhamnose at 1.2-1.4 p.p.m. fall in spectral windows without other peaks, so that the assignment results are certain.

\section{DISCUSSION}

It is commonly assumed that NTPn lack the polysaccharide capsule, the principal pneumococcal virulence factor, which acts by protecting the bacteria during the early phase of infection. Capsule deficiency impairs the capability of NTPn to evade the host's immune defence, so that only rarely are these strains able to persist in the blood and cause invasive disease. The mechanism by which these strains overcome complement activity and phagocytosis during infection remains largely unknown. Other pneumococcal structures, such as the surface proteins PspA (Ren et al., 2003) and PspC (Jarva et al., 2002), are able to inhibit complement activation and deposition, and likely contribute to the inhibition of phagocytosis. Indeed, very few cases of invasive disease due to NTPn have been reported in large epidemiological studies (Beall et al., 2006).

Hathaway and co-workers reported that cps was present in approximately $30 \%$ of naturally occurring NTPn. Nontypability was assumed to be due to mutations in the $c p s$ genes, although sequencing was not performed and the gene(s) involved remained unknown (Hathaway et al., 2004).

In this study, we have investigated the molecular basis of the non-typability of two non-typable strains isolated from cases of bacteraemic pneumonia in the same hospital during the same year. Molecular analysis of the two
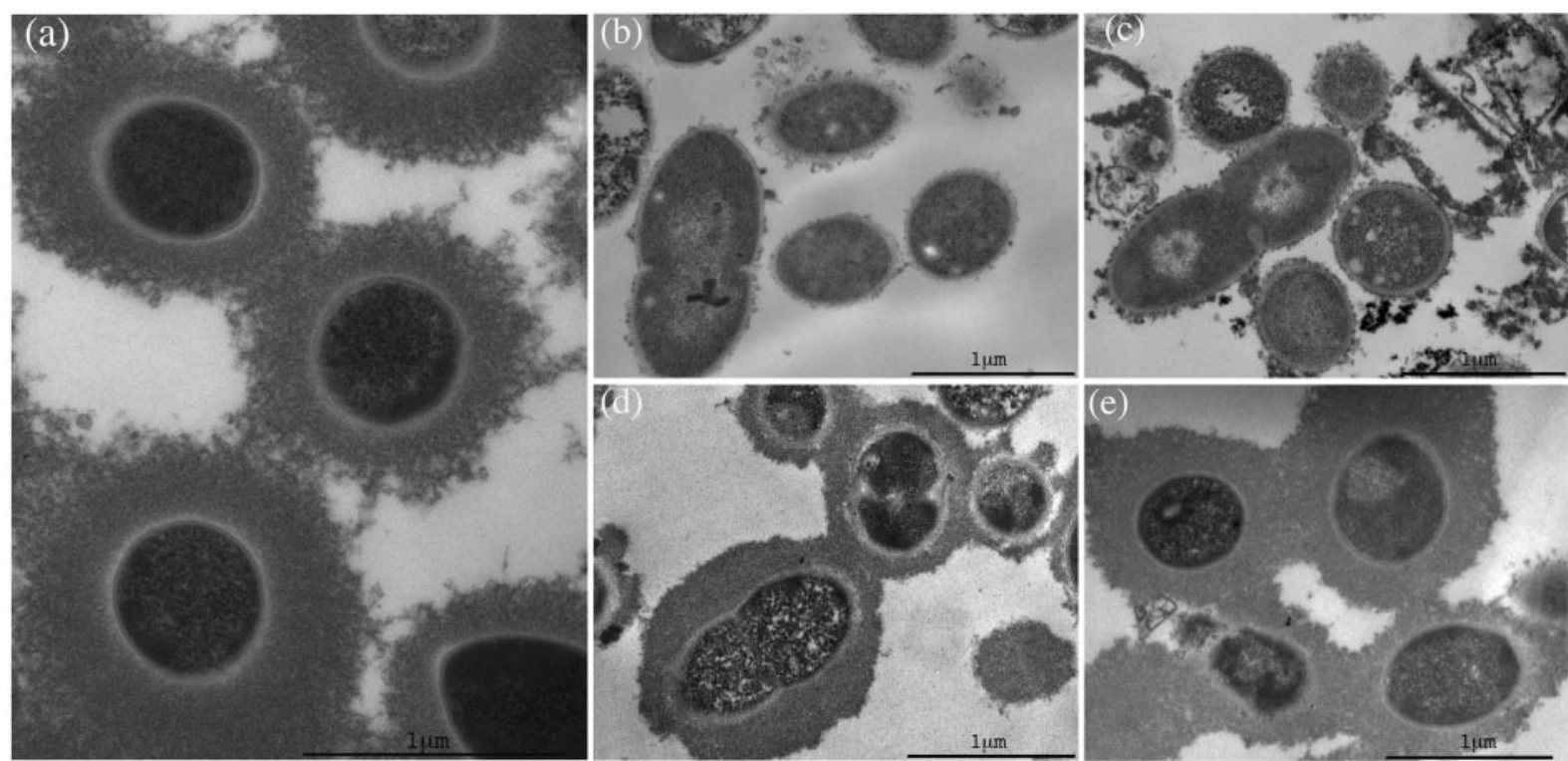

Fig. 2. TEM of S. pneumoniae strains incubated with type 7 antiserum and stained with ruthenium red. (a) AP425 (serotype 7F); (b) AP422 (non-typable); (c) AP426 (non-typable); (d) the WchA-overexpressing AP422 strain; (e) the WchA-overexpressing AP426 strain. Overexpression of WchA restored capsule production in both AP422 and AP426. 


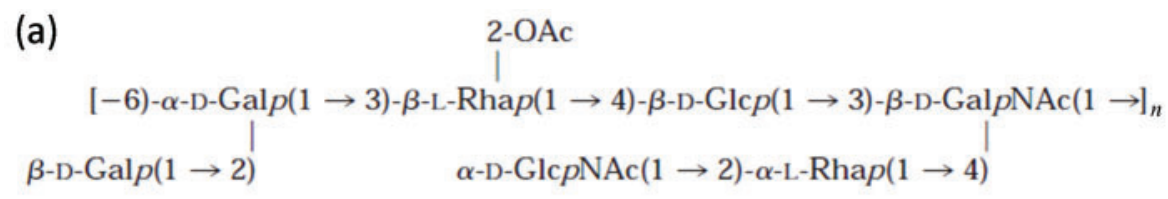

(b)
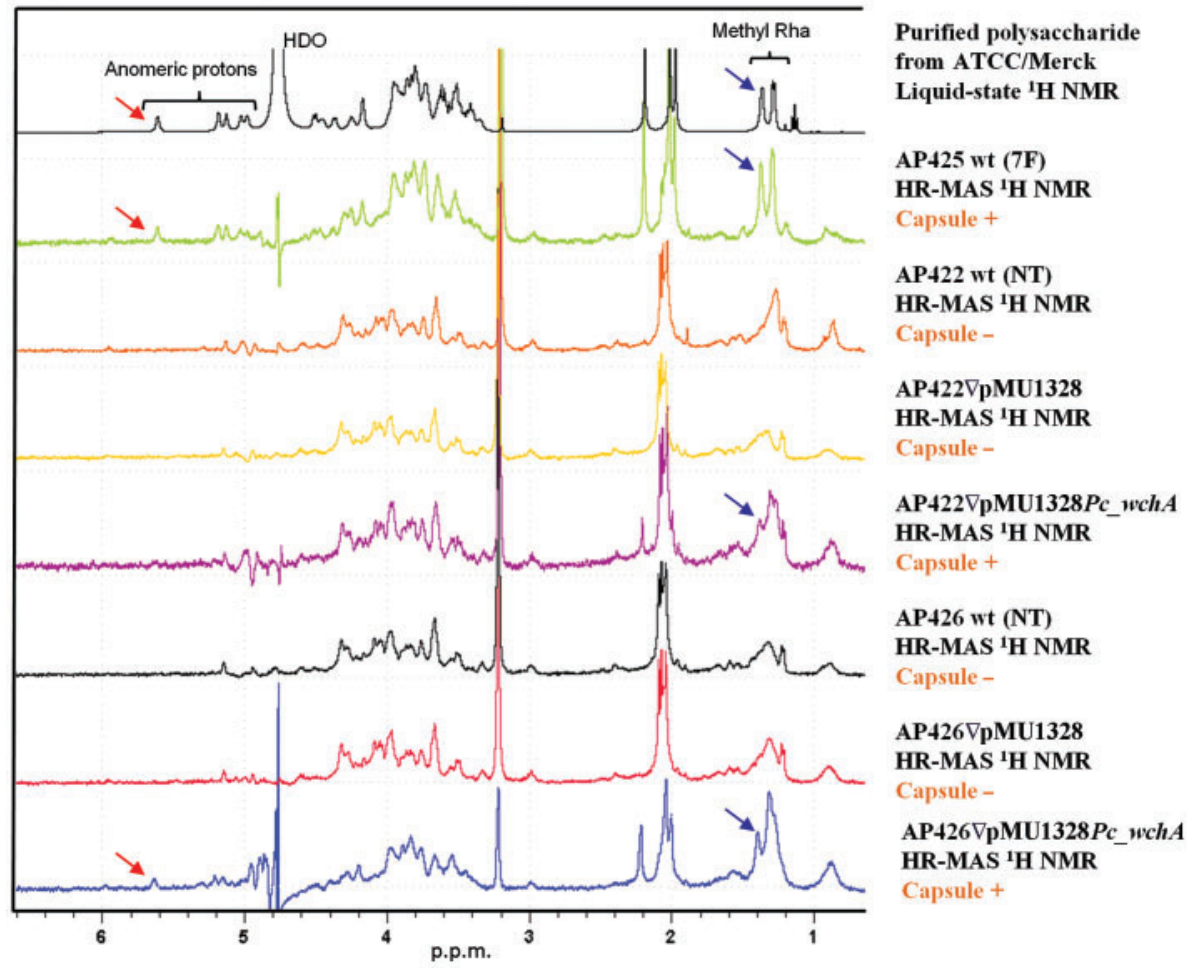

Fig. 3. Proton NMR spectra of purified 7F polysaccharide and S. pneumoniae strains. (a) Schematic representation of the chemical structure of the 7F polysaccharide. (b) Proton HR-MAS NMR spectra recorded in the heterogeneous phase on inactivated bacterial cells. The presence or absence of the capsule on the bacterial surface, based on spectral analysis, is indicated for each strain. Red arrows indicate the anomeric proton at 5.6 p.p.m., while blue arrows indicate the C6 methyl group of rhamnose (Methyl Rha) at 1.2-1.4 p.p.m. Purified 7F polysaccharide solubilized in deuterated water (HDO) was used as a positive control. NT, non-typable; wt, wild-type.

invasive NTPn revealed that the strains were genetically related, since both belonged to ST191 and carried the 7F cps. However, they presented distinct point mutations in the $w c h A$ gene that were responsible for their non-encapsulated phenotype. wchA is responsible for the first step of capsule biosynthesis, since it encodes a glycosyltransferase that catalyses the transfer of the initial sugar to a lipid acceptor, thus initiating the synthesis of the capsule repeat units (Pelosi et al., 2005). Both mutations, introducing a premature stop codon in $w c h A$, are expected to produce a glycosyltransferase truncated in its catalytic domain (Pelosi et al., 2005). Re-establishment of a functional wchA in both NTPn restored the 7F capsule production, as clearly demonstrated by serotyping, TEM and HR-MAS NMR. HR-MAS spectroscopy appears to be a powerful methodology to detect the bacterial capsule and to confirm its identity. In fact, although the polysaccharide capsule content could not be precisely quantified due to the variable number of cells used as an input in the analysis, this method did give a semiquantitative estimate of the specific polysaccharide expressed on the bacterial surface.

Our report is believed to be the first to describe naturally occurring wchA mutations responsible for the non-typable phenotype in pneumococci. The essentiality of wchA for capsular assembly has been shown elsewhere in laboratory mutants of other $S$. pneumoniae serotypes. Insertionduplication mutagenesis or introduction of a premature stop codon in wchA have made pneumococci of serotypes $19 \mathrm{~F}, 14,9 \mathrm{~N}, 13$ and $15 \mathrm{~B}$ unable to produce the capsule (Guidolin et al., 1994; Kolkman et al., 1996, 1998). Furthermore, sequence duplications within wchA have been found to be responsible for capsular phase variation in Sorbarods-generated pneumococci of serotypes 3, 8 and 37 (Waite et al., 2001, 2003). 
This study has identified two new point mutations, both involving $w c h A$, responsible for the unencapsulated phenotype in two NTPn isolated from invasive disease, and this also provides evidence regarding the key role of $w c h A$ in the serotype 7F capsule biosynthesis process. This result suggests that single point mutations represent a mechanism that generates NTPn in nature besides the mechanisms already described, such as downregulation of capsule synthesis or loss of the capsule biosynthetic locus (Hathaway et al., 2004; Hanage et al., 2006). It is intriguing that two genetically related strains, isolated from invasive disease in the same hospital in the same year, underwent distinct point mutations in $w c h A$, thus affecting the production of a type 7F capsule. The success of such strains in causing invasive disease might be due to protection from the immune response exerted by other virulence factors and/or an immune deficiency of the host. Unfortunately, no data are available concerning the immunological status of the patients from whom AP422 and AP426 were isolated. Furthermore, it is difficult to distinguish whether the mutations occurred prior to or during infection, since we do not have serial isolates from the same patient. Extensive analyses of NTPn collections will ascertain whether $w c h A$ is prone to point mutations, leading to the loss of capsule, in other NTPn clinical strains, and estimate how frequently the single point mutation mechanism is used by pneumococci to silence capsule expression.

\section{ACKNOWLEDGEMENTS}

The pMU1328 shuttle plasmid was kindly provided by Intercell AG (Austria). This work was supported in part by a grant from the Italian Ministry of Health (CCM) 'Sorveglianza delle malattie batteriche invasive'. S. M., M. M., F. B. and M. A. B. declare a potential conflict of financial interest as employees of Novartis Vaccines and Diagnostics. The authors have no other competing financial interests.

\section{REFERENCES}

Achen, M. G., Davidson, B. E. \& Hillier, A. J. (1986). Construction of plasmid vectors for the detection of streptococcal promoters. Gene 45, $45-49$.

Alloing, G., Martin, B., Granadel, C. \& Claverys, J. P. (1998). Development of competence in Streptococcus pneumoniae: pheromone autoinduction and control of quorum sensing by the oligopeptide permease. Mol Microbiol 29, 75-83.

Andrade, A. L., Franco, C. M., Lamaro-Cardoso, J., André, M. C. Oliveira, L. L., Kipnis, A., Rocha, C. G., Andrade, J. G., Alves, S. L. \& other authors (2010). Non-typeable Streptococcus pneumoniae carriage isolates genetically similar to invasive and carriage isolates expressing capsular type 14 in Brazilian infants. J Infect 61, 314322.

Arrecubieta, C., López, R. \& García, E. (1994). Molecular characterization of $\operatorname{cap} 3 A$, a gene from the operon required for the synthesis of the capsule of Streptococcus pneumoniae type 3: sequencing of mutations responsible for the unencapsulated phenotype and localization of the capsular cluster on the pneumococcal chromosome. J Bacteriol 176, 6375-6383.
Austrian, R. (1976). The quellung reaction, a neglected microbiologic technique. Mt Sinai J Med 43, 699-709.

Bagnoli, F., Moschioni, M., Donati, C., Dimitrovska, V., Ferlenghi, I., Facciotti, C., Muzzi, A., Giusti, F., Emolo, C. \& other authors (2008). A second pilus type in Streptococcus pneumoniae is prevalent in emerging serotypes and mediates adhesion to host cells. J Bacteriol 190, 5480-5492.

Baldry, S. J., Hinds, J., Gould, K., Lambertsen, L., Hanage, W., Antonio, M., Turner, P., Hermans, P. \& Bentley, S. (2009). Sequencing the cps locus of non-typeable Streptococcus pneumoniae isolates. In $9^{\text {th }}$ European Meeting on the Molecular Biology of the Pneumococcus (Europneumo), Bern, Switzerland.

Beall, B., McEllistrem, M. C., Gertz, R. E., Jr, Wedel, S., Boxrud, D. J., Gonzalez, A. L., Medina, M. J., Pai, R., Thompson, T. A. \& other authors (2006). Pre- and postvaccination clonal compositions of invasive pneumococcal serotypes for isolates collected in the United States in 1999, 2001, and 2002. J Clin Microbiol 44, 999-1017.

Bentley, S. D., Aanensen, D. M., Mavroidi, A., Saunders, D., Rabbinowitsch, E., Collins, M., Donohoe, K., Harris, D., Murphy, L. \& other authors (2006). Genetic analysis of the capsular biosynthetic locus from all 90 pneumococcal serotypes. PLoS Genet 2, e31.

Brown, E. J., Hosea, S. W. \& Frank, M. M. (1983). The role of antibody and complement in the reticuloendothelial clearance of pneumococci from the bloodstream. Rev Infect Dis 5 (Suppl 4), S797-S805.

Calix, J. J. \& Nahm, M. H. (2010). A new pneumococcal serotype, 11E, has a variably inactivated wcjE gene. J Infect Dis 202, 29-38.

Carvalho, M. G., Steigerwalt, A. G., Thompson, T., Jackson, D. \& Facklam, R. R. (2003). Confirmation of nontypeable Streptococcus pneumoniae-like organisms isolated from outbreaks of epidemic conjunctivitis as Streptococcus pneumoniae. J Clin Microbiol 41, 44154417.

Gentile, M. A., Melchiorre, S., Emolo, C., Moschioni, M., Gianfaldoni, C., Pancotto, L., Ferlenghi, I., Scarselli, M., Pansegrau, W. \& other authors (2011). Structural and functional characterization of the Streptococcus pneumoniae RrgB pilus backbone D1 domain. J Biol Chem 286, 14588-14597.

Gherardi, G., D’Ambrosio, F., Monaco, M., Camilli, R., De Florio, L., D'Ancona, F., Dicuonzo, G. \& Pantosti, A. (2009). Population structure of invasive Streptococcus pneumoniae isolates in Italy prior to the implementation of the 7-valent conjugate vaccine (1999-2003). Eur J Clin Microbiol Infect Dis 28, 99-103.

Gladstone, R. A., Jefferies, J. M., Faust, S. N. \& Clarke, S. C. (2011). Continued control of pneumococcal disease in the UK - the impact of vaccination. J Med Microbiol 60, 1-8.

Guidolin, A., Morona, J. K., Morona, R., Hansman, D. \& Paton, J. C. (1994). Nucleotide sequence analysis of genes essential for capsular polysaccharide biosynthesis in Streptococcus pneumoniae type 19F. Infect Immun 62, 5384-5396.

Hanage, W. P., Kaijalainen, T., Saukkoriipi, A., Rickcord, J. L. \& Spratt, B. G. (2006). A successful, diverse disease-associated lineage of nontypeable pneumococci that has lost the capsular biosynthesis locus. J Clin Microbiol 44, 743-749.

Hathaway, L. J., Stutzmann Meier, P., Bättig, P., Aebi, S. \& Mühlemann, K. (2004). A homologue of aliB is found in the capsule region of nonencapsulated Streptococcus pneumoniae. J Bacteriol 186, 3721-3729.

Jacques, M. \& Gottschalk, M. (1997). Use of monoclonal antibodies to visualize capsular material of bacterial pathogens by conventional electron microscopy. Microsc Microanal 3, 234-238.

Jarva, H., Janulczyk, R., Hellwage, J., Zipfel, P. F., Björck, L. \& Meri, S. (2002). Streptococcus pneumoniae evades complement attack and opsonophagocytosis by expressing the $p s p C$ locus-encoded Hic 
protein that binds to short consensus repeats 8-11 of factor $\mathrm{H}$. J Immunol 168, 1886-1894.

Kolkman, M. A., Morrison, D. A., Van Der Zeijst, B. A. \& Nuijten, P. J. (1996). The capsule polysaccharide synthesis locus of Streptococcus pneumoniae serotype 14: identification of the glycosyl transferase gene cps14E. J Bacteriol 178, 3736-3741.

Kolkman, M. A., van der Zeijst, B. A. \& Nuijten, P. J. (1998). Diversity of capsular polysaccharide synthesis gene clusters in Streptococcus pneumoniae. J Biochem 123, 937-945.

Kreikemeyer, B., Gámez, G., Margarit, I., Giard, J. C., Hammerschmidt, S., Hartke, A. \& Podbielski, A. (2011). Genomic organization, structure, regulation and pathogenic role of pilus constituents in major pathogenic streptococci and enterococci. Int $J$ Med Microbiol 301, 240-251.

Llull, D., Muñoz, R., López, R. \& García, E. (1999). A single gene (tts) located outside the cap locus directs the formation of Streptococcus pneumoniae type 37 capsular polysaccharide. Type 37 pneumococci are natural, genetically binary strains. J Exp Med 190, 241-252.

Moreau, M., Richards, J. C., Perry, M. B. \& Kniskern, P. J. (1988). Application of high-resolution n.m.r. spectroscopy to the elucidation of the structure of the specific capsular polysaccharide of Streptococcus pneumoniae type 7F. Carbohydr Res 182, 79-99.

Park, I. H., Pritchard, D. G., Cartee, R., Brandao, A., Brandileone, M. C. \& Nahm, M. H. (2007). Discovery of a new capsular serotype (6C) within serogroup 6 of Streptococcus pneumoniae. J Clin Microbiol $45,1225-1233$.
Pelosi, L., Boumedienne, M., Saksouk, N., Geiselmann, J. \& Geremia, R. A. (2005). The glucosyl-1-phosphate transferase WchA (Cap8E) primes the capsular polysaccharide repeat unit biosynthesis of Streptococcus pneumoniae serotype 8. Biochem Biophys Res Commun 327, 857-865.

Ren, B., Szalai, A. J., Thomas, O., Hollingshead, S. K. \& Briles, D. E. (2003). Both family 1 and family 2 PspA proteins can inhibit complement deposition and confer virulence to a capsular serotype 3 strain of Streptococcus pneumoniae. Infect Immun 71, 75-85.

Sá-Leão, R., Simões, A. S., Nunes, S., Sousa, N. G., Frazão, N. \& de Lencastre, H. (2006). Identification, prevalence and population structure of non-typable Streptococcus pneumoniae in carriage samples isolated from preschoolers attending day-care centres. Microbiology 152, 367-376.

Waite, R. D., Struthers, J. K. \& Dowson, C. G. (2001). Spontaneous sequence duplication within an open reading frame of the pneumococcal type 3 capsule locus causes high-frequency phase variation. $\mathrm{Mol}$ Microbiol 42, 1223-1232.

Waite, R. D., Penfold, D. W., Struthers, J. K. \& Dowson, C. G. (2003). Spontaneous sequence duplications within capsule genes cap $8 E$ and tts control phase variation in Streptococcus pneumoniae serotypes 8 and 37. Microbiology 149, 497-504.

Xu, Q., Kaur, R., Casey, J. R., Sabharwal, V., Pelton, S. \& Pichichero, M. E. (2011). Nontypeable Streptococcus pneumoniae as an otopathogen. Diagn Microbiol Infect Dis 69, 200-204.

Edited by: M. Kilian 